Check for updates

Cite this: RSC Adv., 2017, 7, 39270

\title{
Novel sulfonate-containing halogen-free flame- retardants: effect of ternary and quaternary sulfonates centered on adamantane on the properties of polycarbonate composites $\dagger$
}

\begin{abstract}
Dong Yu Zhu, (D) Jian Wei Guo, * Jia Xing Xian and Shu Qin Fu
In order to find a more environmentally friendly flame retardant, we have designed a novel halogen-free flame-retardant (FR) system consisting of ternary and quaternary sulfonates centered on adamantane. They are named 1,3,5-tri(phenyl-4-sodium sulfonate)adamantane ( $\left.\mathrm{AS}_{3}\right)$ and 1,3,5,7-tetrakis(phenyl-4sodium sulfonate)adamantane $\left(\mathrm{AS}_{4}\right)$, respectively. Both kinds of FRs were synthesized and compounded with polycarbonate (PC) to study their effects on the properties of PC composites. The results show that the new FR system can improve PC flame retardancy efficiently, and has mechanical strength advantages as well. The PC composites with only $0.1 \mathrm{wt} \% \mathrm{AS}_{3}$ or $0.08 \mathrm{wt} \% \mathrm{AS}_{4}$ can pass vertical burning tests (UL94) $\mathrm{V}$-0 level, with increasing the value of limiting oxygen index (LOI) to $31.2 \%$ or $32.3 \%$. Moreover, they can maintain ideal mechanical properties compared to neat PC simultaneously. Finally, the flame retardant mechanism of this system was verified via thermal analysis, morphology and chemical structure analysis of the char residues.
\end{abstract}

Received 12th June 2017

Accepted 3rd July 2017

DOI: $10.1039 / \mathrm{c} 7 \mathrm{ra06504c}$

rsc.li/rsc-advances attracted the interests of more and more researchers from both the academic and industrial fields. ${ }^{9-15}$ However, the general non-halogenated flame retardants (FR) such as phosphorus-, silicon-, and nitrogen-containing FRs are usually not efficient enough to enable PC to pass V-0 rating in the UL 94 test until being incorporated with a relatively large amount. ${ }^{\mathbf{6} 16-20}$ For example, among the above halogen-free FRs, phosphoruscontaining compounds like triphenyl phosphate (TPP) and resorcinol bis(diphenyl phosphate) (RDP) are the most widely used FRs for PC and their blends, but generally, to enable PC passing UL94 V-0 test, the required additive amount should be as much as $5-15 \mathrm{wt} \% .^{21-25}$ As we known, the additive type flame retardants are generally incorporated into polymeric materials by physical means, a large amount of addition may bring in a variety of problems, such as poor transparency, leaching, and a reduction in mechanical properties.

According to the study, ${ }^{\mathbf{2 6 - 2 8}}$ organosulfonates such as the commercially available potassium diphenylsulfone sulfonate (KSS) are highly effective FRs for PC. In general, very low additions (no less than $1 \mathrm{wt} \%$ ) of certain metal salt sulfonates can provide a self-extinguishing performance to polycarbonate. However, the halogen-free flame retarding PC composites incorporated with current sulfonates FRs like KSS still have drawbacks. For example, higher flame retardancy is limited and the mechanical properties suffer a loss, which can be attributed to the poor interfacial adhesion between FR and the polymer matrix. Therefore, to design a series of novel efficient sulfonate FRs and to study the effect of their structure on the properties of
School of Chemical Engineering and Light Industry, Guangdong University of Technology, Guangzhou 510006, China.E-mail: guojw@gdut.edu.cn

$\dagger$ Electronic supplementary information (ESI) available. See DOI: 10.1039/c7ra06504c 
PC composites is of great significance for promoting the development of this field.

In this paper, ternary and quaternary sulfonate centered on adamantane were designed as 1,3,5-tri(phenyl-4-sodium sulfonate)adamantane $\left(\mathrm{AS}_{3}\right)$ and 1,3,5,7-tetrakis(phenyl-4-sodium sulfonate)adamantane ( $\left.\mathrm{AS}_{4}\right)$, respectively. Owing to the various advantages of adamantane such as having stereo-regularity, multiple substitutability, excellent lipophilicity, good thermal and oxidative stabilities and high rigidity, the novel sulfonate flame retardant with high sulfur content is expected to be more efficient for flame retardancy and less negative to mechanical property. In this study, these two kinds of FRs were first synthesized and characterized, then the flammability of PC composites filled with the novel FR were investigated using a cone calorimeter, as well as the LOI and UL-94 tests. The effect of the two kinds of FR content on mechanical properties and thermal properties of $\mathrm{PC}^{-\mathrm{AS}_{n}}(n=3,4)$ was also systematically studied. Finally, the flame retarding mechanism was deduced from the thermal degradation and the residual char morphology and chemical structure. Using this system, PC composites with only $0.08 \mathrm{wt} \% \mathrm{AS}_{4}$ or $0.1 \mathrm{wt} \% \mathrm{AS}_{3}$ can pass V0 and have LOI value higher than 30 , and can maintain ideal mechanical properties as well. In addition, it is found that the higher the sulfonate content in FR molecular structure, the better for flame retardant PC.

\section{Experimental}

\subsection{Materials}

Polycarbonate (PC, grade: 301-10) was supplied by Dow Chemical Company (American) with a melt index (GB 368283) of $20 \mathrm{~g} /$ $10 \mathrm{~min}$ and a density of $0.918 \mathrm{~g} \mathrm{~cm}^{-3}$. PC was dried in the oven at $120{ }^{\circ} \mathrm{C}$ for $8 \mathrm{~h}$ prior to blending. Adamantane (99\%) was purchased from Zhang jia gang Xikai Chemical Co., Ltd., China. Bromine (AR, 99.5\%), Aluminium chloride (AR, 99\%) and tertbutyl bromide (AR, 99\%) were purchased from Aladdin. Chlorosulfonic acid (AR) was supplied by Xiya Reagent Co., Ltd., China. The potassium diphenylsulfone sulfonate (KSS) used here as a reference flame retardant was offered by Guangdong Ever Sun Corp., China. Benzene (AR) and dichloromethane (AR) purchased from Guangzhou Chemical Reagent Factory, were distilled in anhydrous calcium chloride before used. Other chemicals were all used without further purification.

\subsection{Synthesis of ternary and quaternary sulfonate $\mathrm{FR} \mathrm{AS}_{3}$ and $\mathbf{A S}_{4}$}

The $\operatorname{AS}_{n}(n=3,4)$ was synthesized through the reactions of Friedel-crafts arylation from 1-bromoadamantane, ${ }^{29}$ followed by substitution reaction, hydrolysis reaction and neutralization reaction according to the above reaction route as shown in Scheme 1.

A typical procedure for synthesis of 1,3,5-triphenyladamantane is as follows: $5.0 \mathrm{~g}$ 1-bromoadamantane $(23 \mathrm{mmol})$, $6.5 \mathrm{~g}$ tert-butyl bromide ( $50 \mathrm{mmol}$ ), and $175 \mathrm{~mL}$ benzene was introduced into a $500 \mathrm{~mL}$ three-neck flask equipped with a reflux condenser, a calcium chloride drying tube and a magnetic stirrer. After being stirred for $10 \mathrm{~min}$ at $55^{\circ} \mathrm{C}, 0.2 \mathrm{~g}$ aluminium chloride $(1.9 \mathrm{mmol})$ was added slowly and the reaction solution was heated under vigorous reflux for another 10 minutes. Finally, the system was then poured into an ice water and ether mixture, the resultant undissolved substance was filtrated and extracted by chloroform, followed by recrystallization from benzene to get the final product of $1,3,5$ triphenyladamantane.

The procedure for preparing 1,3,5,7-tetraphenyladmantane is very similar to that for 1,3,5-triphenyladamantane, except for using more catalyst of aluminium chloride $(0.6 \mathrm{~g})$ and reacting for a longer time of $60 \mathrm{~min}$ in the same case as shown above. In addition, after the reaction completed, the product is insoluble, so it should be obtained as the filter residue not the filtrate after soxhlet extraction from chloroform. Finally, 1,3,5,7-tetraphenyladmantane was gained after vacuum dried as white solid.

The reaction approaches for both 1,3,5-tri(phenyl-4sulfochloride)adamantane and 1,3,5,7-tetrakis(phenyl-4sulfochloride)adamantane are the same. Take 1,3,5-tri(phenyl4-sulfochloride)adamantane for example, a typical procedure

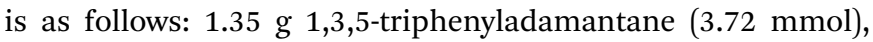
$20 \mathrm{~mL}$ dry dichloromethane was added to a $100 \mathrm{~mL}$ three-neck flask equipped with a reflux condenser, a calcium chloride drying tube, a dropping funnel and a magnetic stirrer in an ice bath, and then to the reaction system was dropwise added $2.5 \mathrm{~mL}$ chlorosulfonic acid and $5 \mathrm{~mL}$ dichloromethane within $10 \mathrm{~min}$. The temperature was slowly raised to $35{ }^{\circ} \mathrm{C}$ and kept stirring for $75 \mathrm{~min}$. After cooling to room temperature, the organic layer was separated by centrifuge, and rotary evaporated to get a brown oil which was washed by water and then filtrated. Finally, $2.35 \mathrm{~g}(95.7 \%)$ solid product of 1,3,5-tri(phenyl-4sulfochloride)adamantane was obtained after vacuum dried at $45{ }^{\circ} \mathrm{C}$ for $12 \mathrm{~h}$.

The procedure for the final step is the same for both $\mathrm{AS}_{3}$ and $\mathrm{AS}_{4}$. To take $\mathrm{AS}_{3}$ for example, a typical method is as follows: $5.0 \mathrm{~g}$ 1,3,5-tri(phenyl-4-sulfochloride)adamantane and $300 \mathrm{~mL}$ deionized water was added to a $500 \mathrm{~mL}$ flask with a reflux condenser and a magnetic stirrer. The mixture was heated at $100{ }^{\circ} \mathrm{C}$ for $24 \mathrm{~h}$ to give a clear solution which was distilled under reduced pressure, and the residue was dried by vacuum oven at $45{ }^{\circ} \mathrm{C}$ for $12 \mathrm{~h}$. Next, the resultant solid was dissolved in $50 \mathrm{~mL}$ of water and was neutralized by $10 \mathrm{wt} \% \mathrm{NaOH}$ solution. After the water was thoroughly removed, the residue was bleached and vacuum dried to afford $5.0 \mathrm{~g}(98.4 \%)$ the product of $\mathrm{AS}_{3}$ as a white salt.

\subsection{Preparation of flame retardant PC composites}

$\mathrm{PC}$ and the flame retardants $\mathrm{AS}_{3}$ and $\mathrm{AS}_{4}$ were first dried overnight at $120{ }^{\circ} \mathrm{C}$ before compounding. The PC composites filled with $0.06-0.16 \mathrm{wt} \% \mathrm{AS}_{3}$ or $\mathrm{AS}_{4}$ FR were prepared in a HL-200 internal mixer (Jilin University Science and Education Instrument factory, China) under $50 \mathrm{rpm}$ for $8 \mathrm{~min}$ at the temperature of $245{ }^{\circ} \mathrm{C}$, which were denoted as $\mathrm{PC} \mathrm{AS}_{3}(0.06 \%), \mathrm{PC} / \mathrm{AS}_{3}$

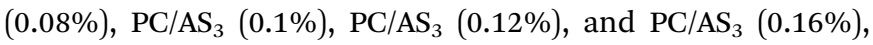
respectively. $\mathrm{PC} / \mathrm{AS}_{4}$ were labeled in the same manner. The compounds were then broken into pieces and dried in a vacuum 

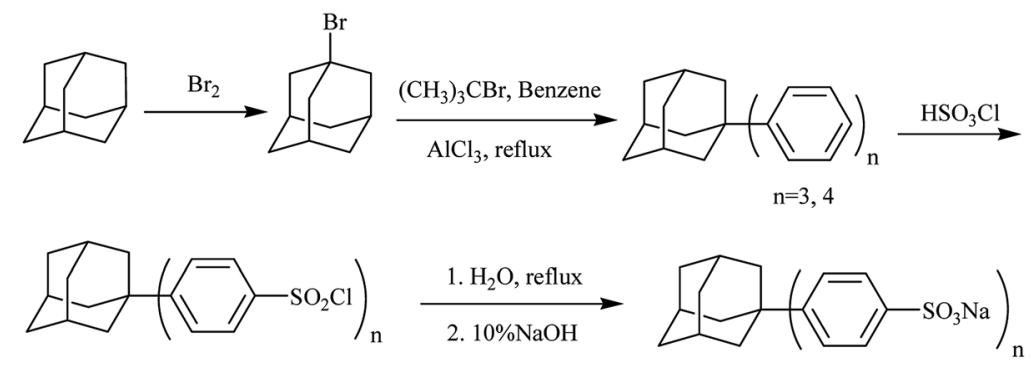

$\mathrm{n}=3,4$

$\mathrm{n}=3,4$
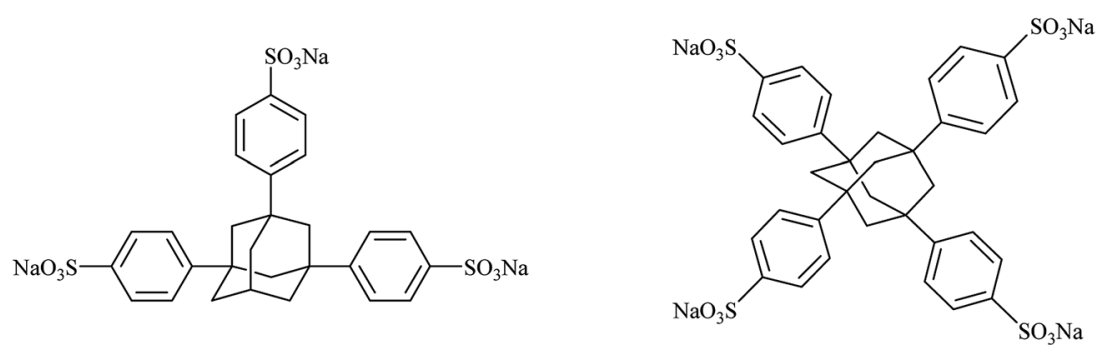

$\mathrm{AS}_{3}$

$\mathrm{AS}_{4}$

Scheme 1 Synthesis route and structures of flame retardants $\operatorname{AS}_{n}(n=3,4)$.

oven at $90{ }^{\circ} \mathrm{C}$ over $5 \mathrm{~h}$. Afterwards, the composite pieces were injection molded or compression molded into standard sizes for different tests. Unfilled PC and the PC composites filled with flame retardant KSS of a suitable amount were also manufactured under the same conditions for comparison.

\subsection{Characterization and measurements}

2.4.1 Spectroscopic analysis. The Fourier transform infrared (FTIR) spectra were recorded with $\mathrm{KBr}$ on a Nicolet 6700 FTIR spectrometer from 400 to $4000 \mathrm{~cm}^{-1}$. The hydrogen nuclear magnetic resonance $\left({ }^{1} \mathrm{H}-\mathrm{NMR}\right)$ spectra were recorded on a Bruker AVANCE III HD400-MHz superconducting NMR spectrometer.

2.4.2 Flammability tests. Limiting oxygen index (LOI) measurements were carried out using a HP6115 type oxygen index test apparatus (Foshan, China) following an ASTM D28632008 standard procedure, and the dimensions of all samples were $80 \mathrm{~mm} \times 10 \mathrm{~mm} \times 4 \mathrm{~mm}$. The LOI values were calculated based on the results of five tests.

The vertical burning tests (UL-94) were conducted on a ZRS-2 vertical horizontal burning apparatus (NEARBYMRO, Germany) according to ASTM D3801 standard, the dimensions of all samples were $125 \mathrm{~mm} \times 13 \mathrm{~mm} \times 3 \mathrm{~mm}$. In the test, five sample bars suspended vertically over surgical cotton were ignited using a $50 \mathrm{~W}$ methane gas burner.

The cone calorimetry test was carried out by using a FTT 0007 cone calorimeter (Fire Testing Technology, UK) in accordance with the ISO 5660 standard procedures. Each specimen, with a dimension of $100 \mathrm{~mm} \times 100 \mathrm{~mm} \times 3 \mathrm{~mm}$, was wrapped in aluminum foil and exposed horizontally to an external heat flux of $35 \mathrm{~kW} \mathrm{~m}{ }^{-2}$. The recorded parameters included the time to ignition (TTI), heat release rate (HRR, $\mathrm{kW} \mathrm{m}^{-2}$ ), peak value of the heat release rate (PHRR, $\mathrm{kW} \mathrm{m}^{-2}$ ), specific extinction area (SEA), the peak value of smoke production rate (SPR), and CO production rate (COP).

2.4.3 Mechanical tests. The tensile and flexural tests were performed using a PHM-5 universal tester according to an ISO 844 and ASTM D790 standard procedure, respectively. Each batch included five specimens to yield averaged values.

\subsection{Thermogravimetric analysis and thermal degradation kinetics}

Thermogravimetric analysis (TGA) and thermal degradation kinetics were carried out with a NetzschSTA409 PC thermoanalyzer instrument (Netzsch, Germany). For the thermal analysis of FRs, each sample was heated from 50 to $800{ }^{\circ} \mathrm{C}$ at the rate of $20{ }^{\circ} \mathrm{C} \mathrm{min}^{-1}$ under nitrogen atmosphere. To test the thermal degradation kinetics, each sample was heated from 50 to $800{ }^{\circ} \mathrm{C}$ at various rates of $5,10,15$ and $20^{\circ} \mathrm{C} \mathrm{min}^{-1}$ under air atmosphere. The Flynn-Wall-Ozawa method was used to analyze the thermal degradation kinetics parameters.

\subsection{Scanning electron microscopy (SEM)}

The SEM observation was performed on a Hitachi S-3400N scanning electron microscope (Hitachi, Japan) to investigate the morphology of the char layer of samples after the LOI tests.

\section{Results and discussion}

\subsection{Chemical structure characterization of FRs}

The flame retardant $\mathrm{AS}_{3}$ and $\mathrm{AS}_{4}$ were synthesized via the reaction route presented in Scheme 1 . The ${ }^{1} \mathrm{H}$ NMR, IR spectra and elementary analysis data for each intermediate product were provided in the ESI (Fig. S1-S7†). Fig. 1(a) shows the ${ }^{1} \mathrm{H}$ 

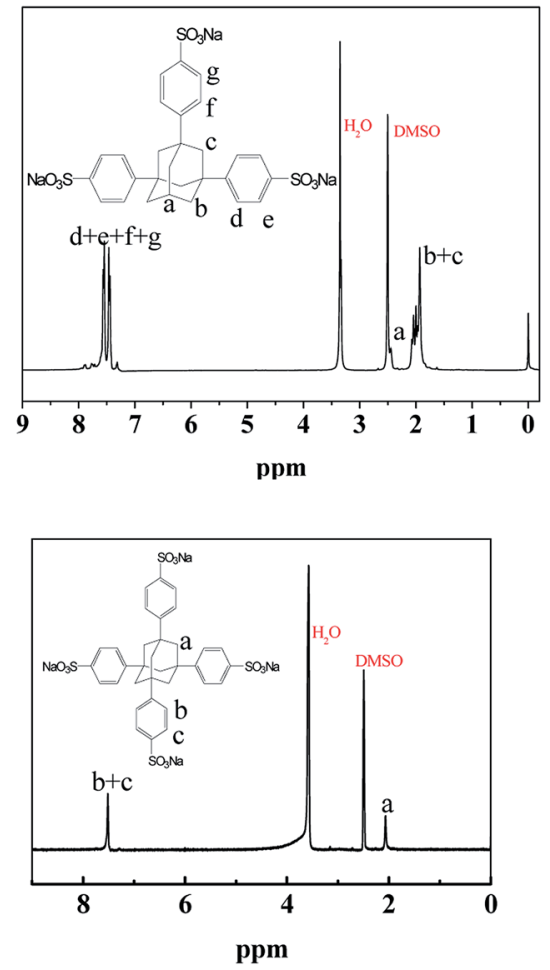

(a)

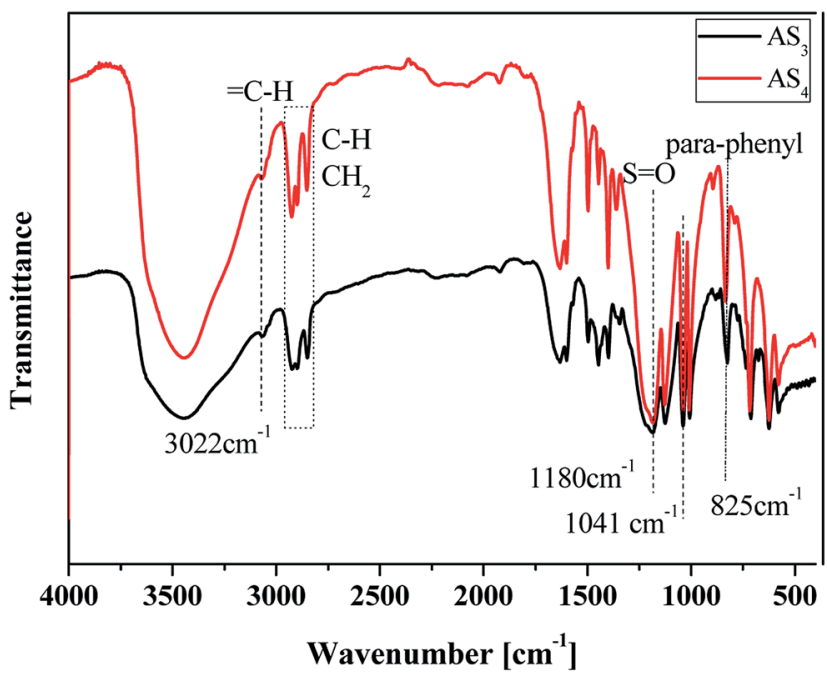

(b)

Fig. 1 (a) ${ }^{1} \mathrm{H}$ NMR spectra of $\mathrm{AS}_{3}$ and $\mathrm{AS}_{4}$ with DMSO- $\mathrm{d}_{6}$ as solvent, (b) FTIR spectra of $\mathrm{AS}_{3}$ and $\mathrm{AS}_{4}$.

NMR spectra of $\mathrm{AS}_{3}$ and $\mathrm{AS}_{4}$ with DMSO-d $\mathrm{d}_{6}$ as a solvent, and Fig. 1(b) the IR spectra.

The ${ }^{1} \mathrm{H}$ NMR spectrum of $\mathrm{AS}_{3}$ is shown in Fig. 1(a), the multipeaks at 1.932-2.070 ppm $(\mathrm{m}, 12 \mathrm{H})$ correspond to the protons $(b+c)$ of $-\mathrm{CH}_{2}$ in adamantyl. The peak at $2.447 \mathrm{ppm}(\mathrm{s}, 1 \mathrm{H})$ is attributed to the (a) proton of $-\mathrm{CH}$ in adamantyl, and the peaks during 7.444-7.564 ppm $(\mathrm{m}, 12 \mathrm{H})$ are due to $(\mathrm{d}+\mathrm{e}+\mathrm{f}+\mathrm{g})$ protons in the benzene ring.
As shown in the right side of Fig. 1(a) which is the ${ }^{1} \mathrm{H}$ NMR spectrum of $\mathrm{AS}_{4}$, we can see the chemical shifts of $\mathrm{H}$ protons at the peak appearing at $2.06(\mathrm{~s}, 12 \mathrm{H})$ correspond to the $\mathrm{H}$ protons (a) in $\mathrm{CH}_{2}$ of tetra-substituted adamantyl, and the peaks at 7.515-7.551 $(\mathrm{m}, 16 \mathrm{H})$ are assigned to $\mathrm{H}$ protons $(\mathrm{b}+\mathrm{c})$ in $=\mathrm{C}-\mathrm{H}$ of benzene ring.

To confirm the successful synthesis of $\mathrm{AS}_{3}$ and $\mathrm{AS}_{4}$, FT-IR spectra were also recorded and presented in Fig. 1(b). $\mathrm{AS}_{3}$ shows almost the same absorption peaks with $\mathrm{AS}_{4}$. The peaks at $3022 \mathrm{~cm}^{-1}, 1600 \mathrm{~cm}^{-1}$ and $1495 \mathrm{~cm}^{-1}$ should be attributed to the stretching vibrations of $=\mathrm{C}-\mathrm{H}$ and $\mathrm{C}=\mathrm{C}$ in benzene rings, respectively, and the characteristic absorption peak of paradisubstituted benzene ring is observed at $825 \mathrm{~cm}^{-1}$. The peaks corresponding to stretching vibration and the deformative vibration of $\mathrm{C}-\mathrm{H}$ and $-\mathrm{CH}_{2}$ in adamantane appear at 2923, 1446, $712 \mathrm{~cm}^{-1}$. The characteristic absorption of $\mathrm{S}=\mathrm{O}$ which belongs to the sulfonate group is observed at 1180 and $1041 \mathrm{~cm}^{-1}$. In addition, it can be also shown that a broad absorption peak appears at $3444 \mathrm{~cm}^{-1}$, which should correspond to the signal of absorbed water. Conclusively, the ${ }^{1} \mathrm{H}$ NMR and the FTIR spectra confirm that $\mathrm{AS}_{3}$ and $\mathrm{AS}_{4}$ have been synthesized successfully.

\subsection{Thermal analysis of FRs}

The basic thermal properties of FRs are vital for their application in polymers.

Fig. 2 shows the thermal stability of the flame retardant $\mathrm{AS}_{3}$ and $\mathrm{AS}_{4}$ by thermogravimetric analysis under nitrogen atmosphere. Both $\mathrm{AS}_{3}$ and $\mathrm{AS}_{4}$ display the similar TG and DTG curves, but $\mathrm{AS}_{4}$ has a little higher degradation temperature. As shown in Fig. 2, about $10 \mathrm{wt} \%$ weight loss between 100 and $300{ }^{\circ} \mathrm{C}$ results from the evaporation of (i) absorbed moisture and (ii) crystal water. According to the weight loss percent of the corresponding part on the curves, the maximum weight loss between $480{ }^{\circ} \mathrm{C}$ and $550{ }^{\circ} \mathrm{C}$ should correspond to the released $\mathrm{SO}_{2}$ from the decomposition of the sulfonate groups, and the last stage of weight loss between $630{ }^{\circ} \mathrm{C}$ and $800{ }^{\circ} \mathrm{C}$ should be attributed to the pyrolysis of -ONa from benzene rings. The residues should be 1,3,5-triphenyladamantane or 1,3,5,7-tetraphenyladamantane and
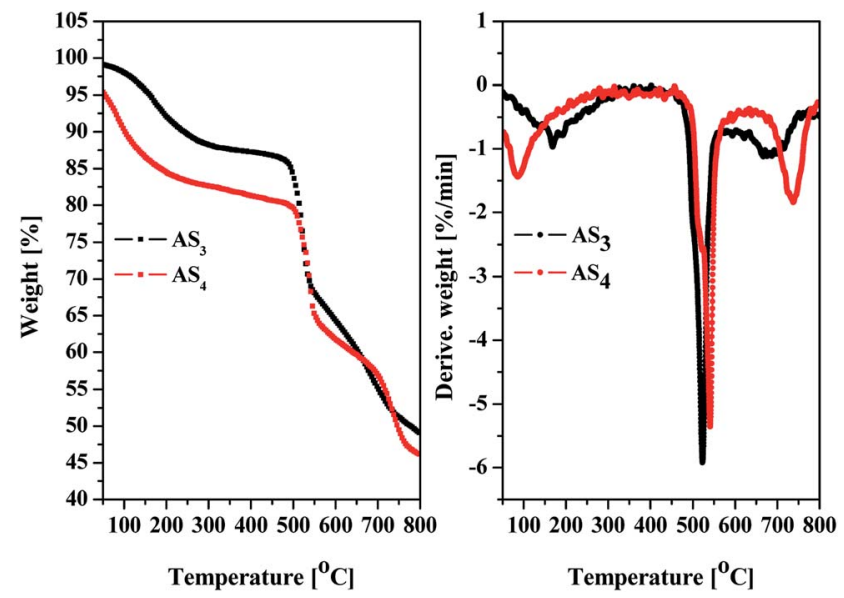

Fig. 2 TG and DTG curves of $\mathrm{AS}_{3}$ and $\mathrm{AS}_{4}$ recorded in $\mathrm{N}_{2}$. 
sodium ions. In general, proper decomposition temperature is very important for additive-type flame retardants to work for matrix materials. The maximum decomposition peak temperature which corresponds to the degradation of sulfonate is $527.7{ }^{\circ} \mathrm{C}$ for $\mathrm{AS}_{3}$ and $543.3{ }^{\circ} \mathrm{C}$ for $\mathrm{AS}_{4}$, that is much higher than the thermo-processing temperature of PC composites and matchable with the degradation temperature of PC $\left(T_{\text {peak }}=\right.$ 534.2 ${ }^{\circ} \mathrm{C}$ ) (see Fig. 7). It demonstrates that the novel FR system of $\mathrm{AS}_{3}$ and $\mathrm{AS}_{4}$ can well meet the demand for flame retardants of $\mathrm{PC}$ in regards to thermal property.

\subsection{Flammability of PC composites}

The flammability of $\mathrm{PC} / \mathrm{AS}_{n}(n=3,4)$ composites was systematically evaluated using LOI, UL-94 and cone calorimetry methods. The results of LOI and UL-94 tests for $\mathrm{PC} / \mathrm{AS}_{n}$ with different loadings and PC/KSS composites as the control samples are displayed in the above Fig. 3. As shown in Fig. 3, the LOI value of neat PC is $25.8 \%$. After being added with the synthesized new FRs, we can see that both $\mathrm{AS}_{3}$ and $\mathrm{AS}_{4}$ improved the flammability of PC composites efficiently. According to the JISK 7201 industrial standard, with LOI $>30 \%$, $\mathrm{PC} / \mathrm{AS}_{3}$ with $\mathrm{AS}_{3}$ content between 0.1-0.16 wt\% and PC/AS 4 with $\mathrm{AS}_{4}$ content $0.08-0.16 \mathrm{wt} \%$ within the range studied, can all be assigned to the first class flame retardant materials that are extremely hard to ignite. As to the UL-94 tests, it also can be clearly indicated from Fig. 3 that the UL-94 rating for neat PC is $\mathrm{V}-2$. As to the flame retardant PC composites, only $0.1 \mathrm{wt} \% \mathrm{AS}_{3}$ or even $0.08 \mathrm{wt} \% \mathrm{AS}_{4}$ can enhance $\mathrm{PC}$ materials to pass $\mathrm{V}$ 0 rating. So the optimal FR addition amount can be $0.1 \mathrm{wt} \%$ for $\mathrm{AS}_{3}$ and $0.08 \mathrm{wt} \%$ for $\mathrm{AS}_{4}$. In contrast, the control samples, which are added with KSS as FR instead, under the same FR content, can only reach V-2 with the LOI value of $27.1 \%$ for PC/ KSS $(0.08 \mathrm{wt} \%)$ and $28.6 \%$ for PC/KSS (0.1 wt $\%)$, respectively. Thus the above results from both LOI and UL-94 manifest that our synthesized $\mathrm{AS}_{3}$ or $\mathrm{AS}_{4}$ indeed works well in flame-retarding of PC composites, and $\mathrm{AS}_{4}$ which has more sulfonate content in molecular structure is shown more efficient than $\mathrm{AS}_{3}$.

Cone calorimetry, as one of the most important bench-scale methods for evaluating the flame retarding performance of materials in a real fire case ${ }^{30}$ was further investigated in this

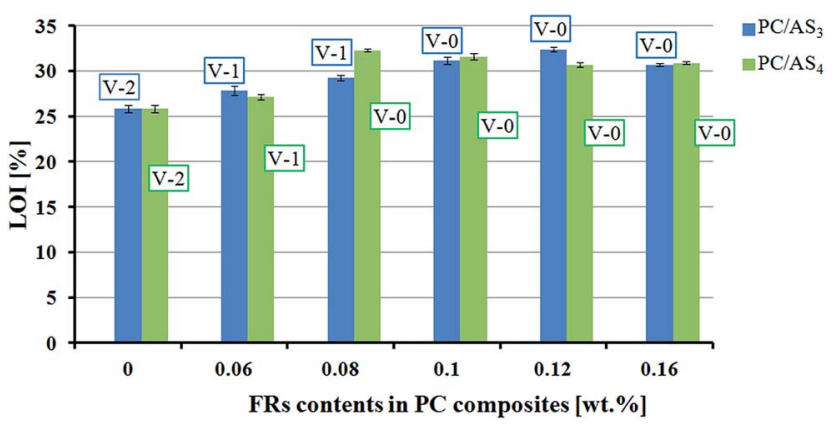

Fig. 3 The LOI values and UL-94 results of flame retardant PC composites $\mathrm{PC}_{\mathrm{AS}}$ and $\mathrm{PC} / \mathrm{AS}_{4}$ with different $\mathrm{FR}$ contents. For the control sample of PC/KSS $(0.08 \%)$ is $27.1 \%, \mathrm{~V}-2$, and PC/KSS $(0.1 \%)$ $28.6 \%, \mathrm{~V}-2$.
Table 1 Cone calorimetric data with a heat flux of $35 \mathrm{~kW} \mathrm{~m}^{-2}$

\begin{tabular}{|c|c|c|c|}
\hline Sample & $\mathrm{PC}$ & $\mathrm{PC} \mathrm{AS}_{3}(0.10 \%)$ & $\mathrm{PC} / \mathrm{AS}_{4}(0.08 \%)$ \\
\hline TTI/s & 102 & 72 & 86 \\
\hline pHRR $\left(\mathrm{kW} \mathrm{m}^{-2}\right)$ & 305.9 & 243.2 & 247.8 \\
\hline THR $\left(\mathrm{MJ} \mathrm{m}^{-2}\right)$ & 61.0 & 53.3 & 59.4 \\
\hline SEA $\left(\mathrm{m}^{3} \mathrm{~kg}^{-1}\right)$ & 680.5 & 510.3 & 573.3 \\
\hline $\operatorname{pkSPR}\left(\mathrm{m}^{3} \mathrm{~s}^{-1}\right)$ & 0.133 & 0.081 & 0.079 \\
\hline Mean COY $\left(\mathrm{kg} \mathrm{kg}^{-1}\right)$ & 0.148 & 0.103 & 0.089 \\
\hline
\end{tabular}

work. The samples of $\mathrm{PC} / \mathrm{AS}_{3}(0.1 \%), \mathrm{PC} / \mathrm{AS}_{4}(0.08 \%)$ and $\mathrm{PC}$ are tested by cone calorimeter and some of the important results are summarized in Table 1. The heat release rate (HRR), in particular the peak of HRR (pHRR) value, is proved to be the most useful parameter to evaluate fire safety. Fig. 4 shows the HRR plots for pure $\mathrm{PC}, \mathrm{PC} / \mathrm{AS}_{3}(0.1 \%)$, and $\mathrm{PC} / \mathrm{AS}_{4}(0.08 \%)$ composites.

It can be seen from Table 1 and Fig. 4 that neat PC shows a very sharp HRR curve at the time range of 100-400 s with a maximum of $305.9 \mathrm{~kW} \mathrm{~m}^{-2}$ at $125 \mathrm{~s}$. In comparison, the HRR curves of $\mathrm{PC} / \mathrm{AS}_{3}(0.1 \%)$ and $\mathrm{PC} / \mathrm{AS}_{4}(0.08 \%)$ both become flatter, indicating the lower intensity of a fire. The peak HRR (PHRR) values decrease to $243.2 \mathrm{~kW} \mathrm{~m}^{-2}$ and $247.8 \mathrm{~kW} \mathrm{~m}^{-2}$ for $\mathrm{PC} / \mathrm{AS}_{3}$ $(0.1 \%)$ and $\mathrm{PC} / \mathrm{AS}_{4}(0.08 \%)$, respectively. The total heat release (THR) value for neat $\mathrm{PC}$ is $61.0 \mathrm{MJ} \mathrm{m}^{-2}$, and it also decreases to $53.3 \mathrm{MJ} \mathrm{m}^{-2}$ and $59.4 \mathrm{MJ} \mathrm{m}{ }^{-2}$ for $\mathrm{PC} / \mathrm{AS}_{3}(0.1 \%)$ and $\mathrm{PC} / \mathrm{AS}_{4}$ $(0.08 \%)$, respectively. These results demonstrate that both $\mathrm{AS}_{3}$ and $\mathrm{AS}_{4}$ can effectively reduce the risk of fire hazard.

Smoke and toxic gas release performances are other very important parameters to determine flame-retardant material in fire safety judgment. ${ }^{31}$ As seen in Table 1 , the SEA data which measures the total generated smoke quantity decreases from $680.5 \mathrm{~m}^{3} \mathrm{~kg}^{-1}$ for PC to $510.3 \mathrm{~m}^{3} \mathrm{~kg}^{-1}$ and $573.3 \mathrm{~m}^{3} \mathrm{~kg}^{-1}$ for $\mathrm{PC} / \mathrm{AS}_{3}(0.1 \%)$ and $\mathrm{PC} / \mathrm{AS}_{4}(0.08 \%)$, respectively. And the pkSPR is also decreased from $0.133 \mathrm{~m}^{3} \mathrm{~s}^{-1}$ to $0.081 \mathrm{~m}^{3} \mathrm{~s}^{-1}$ and $0.079 \mathrm{~m}^{3} \mathrm{~s}^{-1}$, respectively. The lower value of SEA and pkSPR indicates that $\mathrm{AS}_{3}$ and $\mathrm{AS}_{4}$ can both strongly cut down the

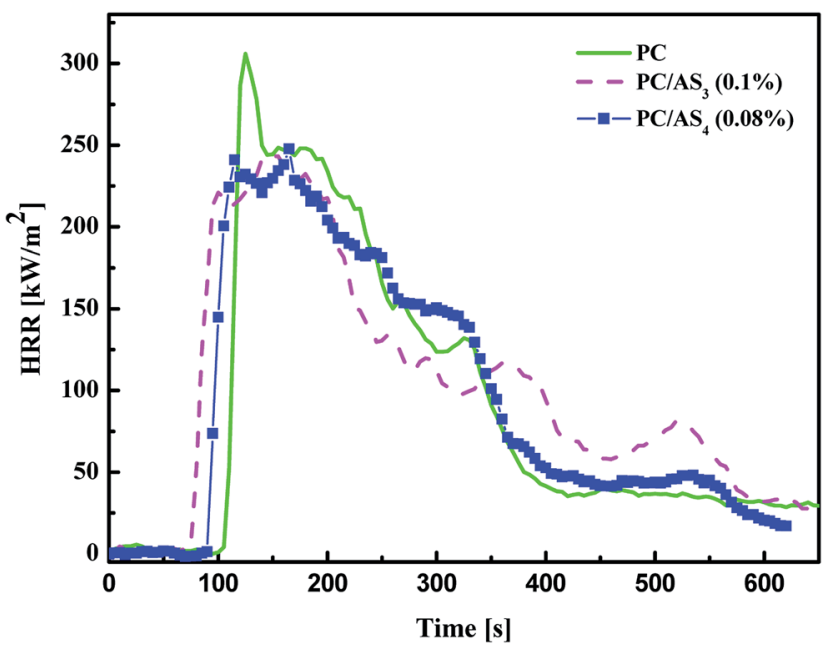

Fig. 4 Heat release rate curves of $\mathrm{PC}, \mathrm{PC} \mathrm{AS}_{3}(0.1 \%)$ and $\mathrm{PC} / \mathrm{AS}_{4}$ $(0.08 \%)$ composites. 


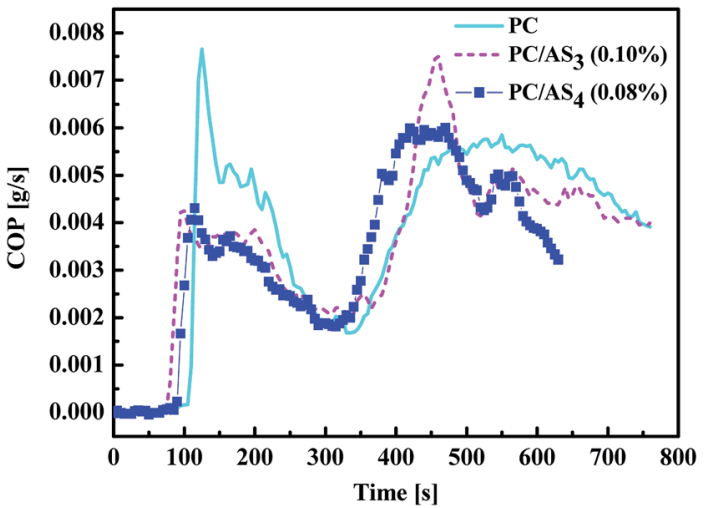

Fig. 5 CO release rate curves of $\mathrm{PC}, \mathrm{PC} / \mathrm{AS}_{3}(0.1 \%)$ and $\mathrm{PC} / \mathrm{AS}_{4}(0.08 \%)$ composites.

smoke emission of PC composites. Fig. 5 shows the variation of $\mathrm{CO}$ production during the combustion process. The mean $\mathrm{CO}$ yield of PC is $0.148 \mathrm{~kg} \mathrm{~kg}^{-1}$. With addition of $\operatorname{AS}_{n}(n=3,4) \mathrm{FR}$, the mean CO yield decreased to $0.103 \mathrm{~kg} \mathrm{~kg}^{-1}$ and $0.089 \mathrm{~kg} \mathrm{~kg}^{-1}$ for $\mathrm{PC} / \mathrm{AS}_{3}(0.1 \%)$ and $\mathrm{PC} / \mathrm{AS}_{4}(0.08 \%)$, respectively. Moreover, the first peak $\mathrm{CO}$ production is significantly decreased from $0.0077 \mathrm{~kg} \mathrm{~kg}^{-1}$ for neat PC to $0.0043 \mathrm{~kg} \mathrm{~kg}^{-1}$ and $0.0044 \mathrm{~kg} \mathrm{~kg}^{-1}$, respectively. This means that the $\mathrm{AS}_{3}$ or $\mathrm{AS}_{4}$ flame retardant system can efficiently suppress poisonous gas emission so as to increase the likelihood of survival at the beginning of a fire.

It is worthwhile to mention that the TTI of pure PC is $102 \mathrm{~s}$, while it is decreased to $72 \mathrm{~s}$ and $86 \mathrm{~s}$ with addition of $0.1 \mathrm{wt} \%$ $\mathrm{AS}_{3}$ and $0.08 \mathrm{wt} \% \mathrm{AS}_{4}$, respectively. The unexpected decrease in TTI of $\mathrm{PC} / \mathrm{AS}_{n}$ composites indicates that $\mathrm{AS}_{3}$ and $\mathrm{AS}_{4}$ may retard the combustion of PC matrix through accelerating the thermal decomposition of PC and quickly promoting the formation of char layers at relatively lower temperature.

From the LOI test, UL-94 test and cone calorimeter results which are discussed above, it is clear that the flame retardancy of PC is significantly enhanced by incorporation of $\mathrm{AS}_{3}$ or $\mathrm{AS}_{4}$ FRs.

\subsection{Mechanical properties}

As an additive-type flame retardant, the effect of its incorporation on the mechanical properties of matrix material is a matter of concern. Fig. 6 depicts the tensile and flexural properties of PC and its composites. As shown in Fig. 6(a), the tensile strength of virgin $\mathrm{PC}$ is $60.55 \mathrm{MPa}$. With increasing of $\mathrm{AS}_{3}$ content, the tensile strength of $\mathrm{PC} / \mathrm{AS}_{3}$ composites increases first and then levels off after a slight decrease. As to PC/AS 4 , the tensile strength keeps gradually increasing and then levels off with additional $\mathrm{AS}_{4}$ content. With the addition of $\mathrm{FR}$ at the optimal content for flame retardancy, the tensile strength rises to $61.58 \mathrm{MPa}$ and $61.88 \mathrm{MPa}$ for $\mathrm{PC} / \mathrm{AS}_{3}(0.1 \%)$ and $\mathrm{PC} / \mathrm{AS}_{4}$ $(0.08 \%)$, respectively. While the control samples of $\mathrm{PC} / \mathrm{KSS}$ $(0.1 \%)$ and $\mathrm{PC} / \mathrm{KSS}(0.08 \%)$ have the tensile strength of 59.88 MPa and 60.1 MPa, respectively, which are both lower than that of neat PC. As to the flexural test, we see that the flexural strength of $\mathrm{PC} / \mathrm{AS}_{3}$ is slightly decreasing and then increasing with the rise of $\mathrm{AS}_{3}$ content, while for $\mathrm{PC} / \mathrm{AS}_{4}$, the
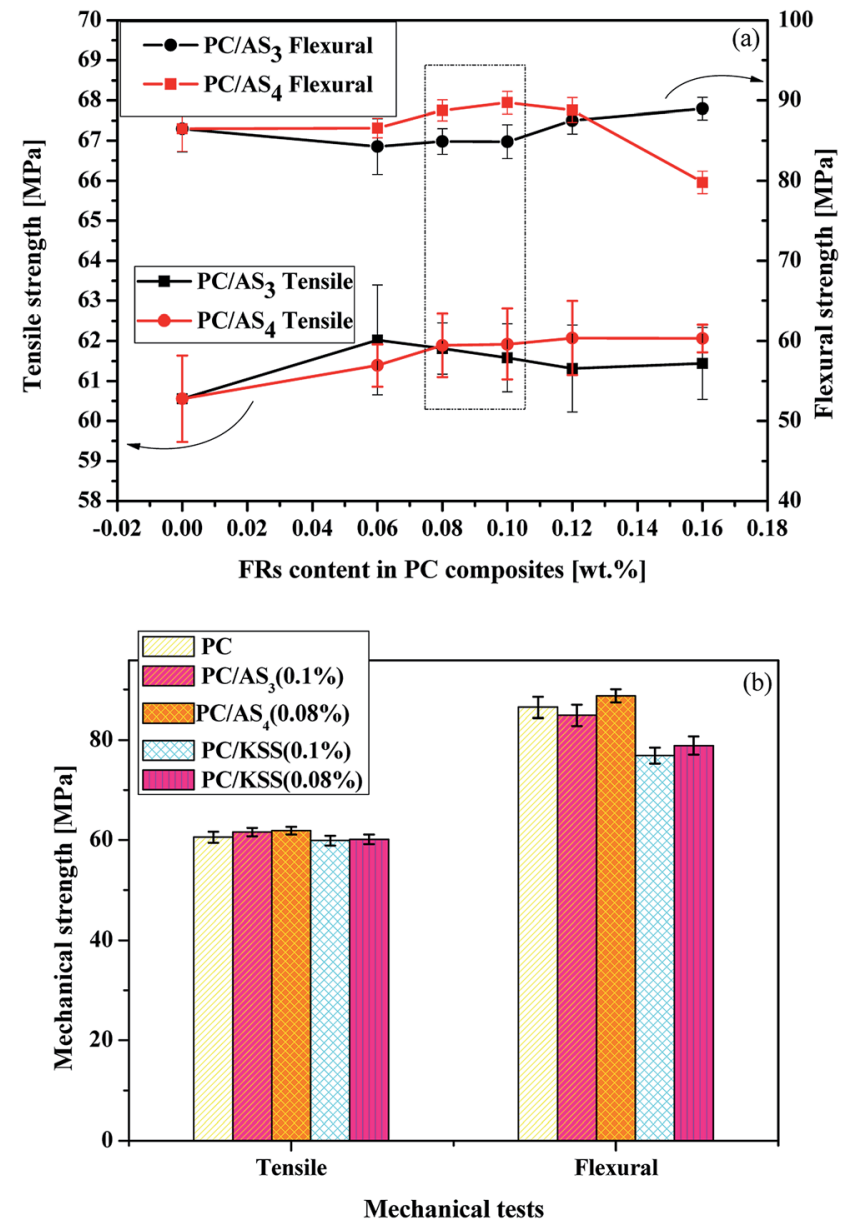

Fig. 6 (a) Effect of the FR content on the mechanical strengths of PC/ $\mathrm{AS}_{3}$ and $\mathrm{PC} / \mathrm{AS}_{4}$ composites; (b) histogram of the mechanical strengths of $\mathrm{PC}, \mathrm{PC} / \mathrm{AS}_{3}(0.1 \%), \mathrm{PC}^{-\mathrm{AS}_{4}}(0.08 \%)$ and the control samples of $\mathrm{PC} /$ KSS $(0.1 \%)$ and PC/KSS $(0.08 \%)$

flexural strength increases first and then decreases sharply. At the optimal addition of each FR for flame retardancy, the flexural strength of $\mathrm{PC} / \mathrm{AS}_{3}(0.1 \%)$ is $84.87 \mathrm{MPa}$, slightly reduced by $1.8 \%$ compared to that of pure $\mathrm{PC}$ of $86.47 \mathrm{MPa}$, and $\mathrm{PC} / \mathrm{AS}_{4}$ $(0.08 \%)$ is $88.75 \mathrm{MPa}$, increased by $2.6 \%$ compared to that of PC. In contrast, the flexural strengths are only $76.88 \mathrm{MPa}$ and 78.88 MPa for the control samples of PC/KSS (0.1\%) and PC/KSS $(0.08 \%)$, respectively, which is decreased by $11 \%$ than PC.

In conclusion, the novel FR system we proposed in this study can effectively alleviate the deterioration of FR to the mechanical strength of polymeric matrix, and can even improve it to a certain extent in some cases. The advantages of $\mathrm{AS}_{3}$ and $\mathrm{AS}_{4} \mathrm{FR}$ over the current existing control FR for PC could be attributed to the low incorporated content and the high stiffness and lipid solubility of adamantly that make $\mathrm{AS}_{3}$ and $\mathrm{AS}_{4}$ of better compatibility with PC.

\subsection{Flame retardant mechanism}

3.5.1 Thermal decomposition properties of PC composites. In order to clearly understand the flame retardant mechanism of $\mathrm{PC} / \mathrm{AS}_{3}$ and $\mathrm{PC} / \mathrm{AS}_{4}$, the thermal stability of the flame 
retardant PC composites is investigated by TGA. Moreover, the Flynn-Wall-Ozawa method is used to analyze their thermal degradation kinetics.

Histogram of average activation energy of $\mathrm{PC}, \mathrm{PC} \mathrm{AS}_{3}(0.1 \%)$, and $\mathrm{PC} / \mathrm{AS}_{4}(0.08 \%)$ with the degradation conversion rate from 0 to $70 \%$ calculated by the Flynn-Wall-Ozawa method.

The thermal degradation and stability of polymers have substantial connection with their flame retardancy. Therefore, TG analysis was used to investigate the thermal degradation properties of PC and its composites. Fig. 7(a) shows the TG and DTG curves under air for the PC, $\mathrm{PC} / \mathrm{AS}_{3}(0.1 \%)$, and $\mathrm{PC} / \mathrm{AS}_{4}$ $(0.08 \%)$ composites, respectively. Compared to $\mathrm{PC}$, it is observed that both the TG and DTG curves of PC/AS $3(0.1 \%)$ and $\mathrm{PC} / \mathrm{AS}_{4}(0.08 \%)$ shift forward to lower temperature successively. The corresponding data such as onset decomposition temperature $\left(T_{5 \%}\right)$, the temperature at maximum weight loss rate $\left(T_{\text {peak }}\right)$ are list in the insert table of Fig. 7(a). It is found that the $T_{5 \%}, T_{\text {peak }}$ of PC are $485.2{ }^{\circ} \mathrm{C}$ and $534.2{ }^{\circ} \mathrm{C}$, respectively. However, the $T_{5 \%}, T_{\text {peak }}$ are $468.2{ }^{\circ} \mathrm{C}, 528.8{ }^{\circ} \mathrm{C}$, and $470.4{ }^{\circ} \mathrm{C}$, $521.9^{\circ} \mathrm{C}$ for $\mathrm{PC} / \mathrm{AS}_{3}(0.1 \%)$ and $\mathrm{PC} / \mathrm{AS}_{4}(0.08 \%)$ respectively. This result is in accordance with the variation of TTI listed in Table 1 that the TTI of the PC/AS 3 and PC/AS 4 composites are shorter than that of virgin PC. It can be deduced that the sulfonate groups in $\mathrm{AS}_{3}$ or $\mathrm{AS}_{4}$ flame retardants accelerate the
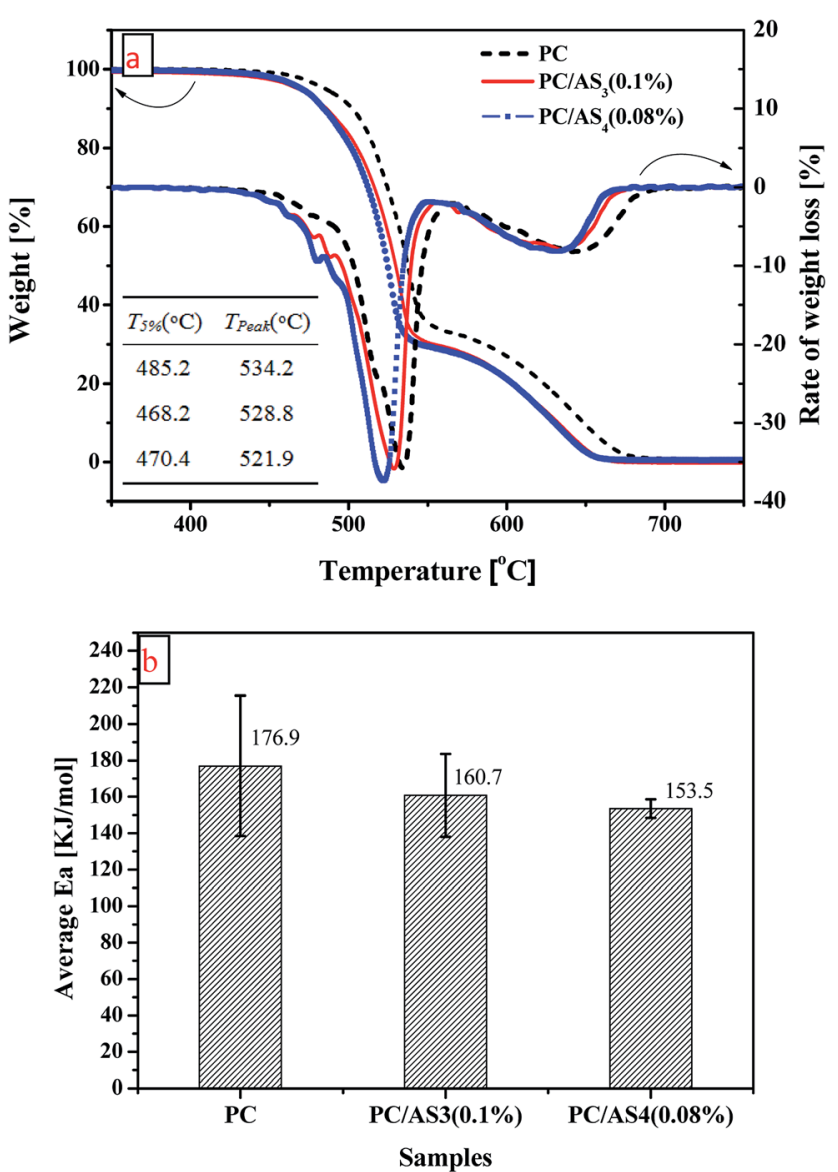

Fig. 7 (a) TG and DTG curves of PC, $P C / \mathrm{AS}_{3}(0.1 \%), \mathrm{PC} \mathrm{AS} \mathrm{S}_{4}(0.08 \%)$ composites, (b). decomposition of $\mathrm{PC}$ and promote its char-forming so as to result in the decline of the degradation temperature as well as TTI. Therefore, the decreased thermal stability is essential rather than a drawback of the multi-sulfonate FR system.

Furthermore, to study the degradation behavior of the PC composites, the Flynn-Wall-Ozawa method is used to analyze their thermal degradation kinetics. According to this method, the degradation activation energy can be determined directly from the $\log \beta$ versus $1 / T$ plot as follows. ${ }^{32}$

$$
\log f(\alpha)=\log \frac{A E}{R}-\log \beta-2.315-0.4567 \frac{E}{R T}
$$

where $A$ is the pre-exponential factor, $R$ is the gas constant, $E$ is the activation energy, $\beta$ is heating rate, $T$ is the Kelvin temperature, and $f(\alpha)$ is the integrated form of the conversion dependence function. The TG curves for PC, $\mathrm{PC} / \mathrm{AS}_{3}(0.1 \%), \mathrm{PC} / \mathrm{AS}_{4}$ $(0.08 \%)$ composites recorded under various heating flow rates of $5,10,15$ and $20{ }^{\circ} \mathrm{C} \mathrm{min}^{-1}$ under air atmosphere are shown in Fig. S8-S10. $\dagger$ The activation energy data of the three samples calculated by the Flynn-Wall-Ozawa method are listed in Tables S1-S3, $\dagger$ and the average thermal degradation activation energies within the conversion rate from 0 to $70 \%$ are shown in Fig. 7(b). It can be seen clearly from Fig. 7(b) that the average activation energy of $\mathrm{PC}$ is $176.9 \mathrm{~kJ} \mathrm{~mol}^{-1}$. When $\mathrm{AS}_{3}$ or $\mathrm{AS}_{4}$ is added, the average activation energy decreases to $160.7 \mathrm{~kJ} \mathrm{~mol}^{-1}$ and $153.5 \mathrm{~kJ} \mathrm{~mol}^{-1}$ for $\mathrm{PC}^{-\mathrm{AS}_{3}}(0.1 \%)$ and $\mathrm{PC} / \mathrm{AS}_{4}(0.08 \%)$, respectively. Comparatively, the average activation energy of $\mathrm{PC} /$ $\mathrm{AS}_{4}(0.08 \%)$ is lower than that of $\mathrm{PC}^{-\mathrm{AS}_{3}}(0.1 \%)$, which demonstrates stronger accelerating decomposition. This result further manifests that both $\mathrm{AS}_{3}$ and $\mathrm{AS}_{4}$ can accelerate $\mathrm{PC}$ decomposition. $\mathrm{AS}_{4}$, with more sulfonate content in its molecular structure is relatively stronger, which is consistent with TGA results as shown in Fig. 7(a). This could be due to the $\mathrm{SO}_{2}$ generated from sulfonate degradation which shows high catalysis ability for PC decomposition.

3.5.2 Morphology and structure analysis of char residue. In order to further understand the flame retarding mechanism of $\mathrm{PC} / \mathrm{AS}_{n}$ system, the morphology and chemical structure of residual char obtained from the sample after LOI test was investigated by SEM and FT-IR, respectively. As can be observed in Fig. 8, the residue char surface of PC is smooth but with macro-pores and crevices, which cannot provide a good barrier for the polymer. In comparison, the char layers of both $\mathrm{PC} / \mathrm{AS}_{3}$ and $\mathrm{PC} / \mathrm{AS}_{4}$ samples are continuous and expansionary with a multitude of expanded spheres. This intumescent char layer provides a good flame shield to stop the propagation of heat and mass to the underneath layer, and delays the volatilization of combustible gases, it also retards the penetration of oxygen, and the feedback of heat. Consequently, such a useful char layer as the flame barrier can make the corresponding PC composites achieve excellent fire retardation.

Fig. 9 shows the FT-IR spectra of virgin PC and the residual char of $\mathrm{PC} / \mathrm{AS}_{3}(0.1 \%)$ and $\mathrm{PC} / \mathrm{AS}_{4}(0.08 \%)$, respectively. We can see that the FT-IR spectra for residual char of $\mathrm{PC} / \mathrm{AS}_{3}$ and $\mathrm{PC} / \mathrm{AS}_{4}$ are almost the same, but are of many differences compared to that of the virgin PC. The absorption peak in $3439 \mathrm{~cm}^{-1}$ and $1400 \mathrm{~cm}^{-1}$ represents the stretching and plane bending 

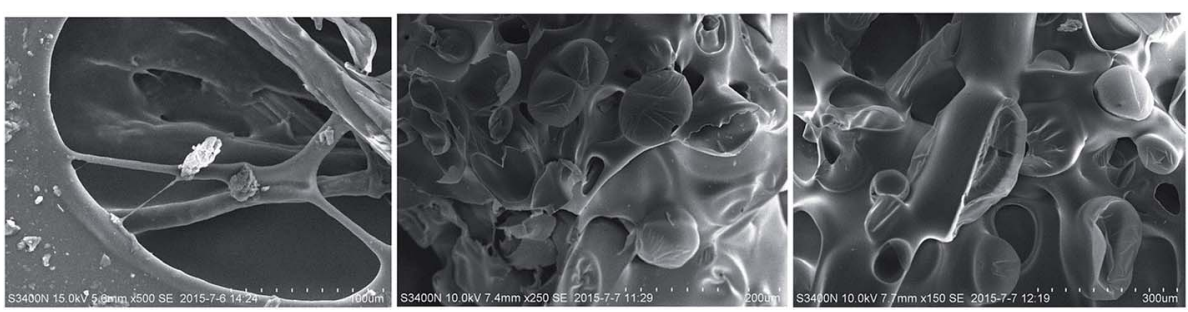

Fig. 8 SEM photos of the residue char for (a) PC, (b) PC/AS $3(0.1 \%)$ and (c) $P C / \mathrm{AS}_{4}(0.08 \%)$ composites.

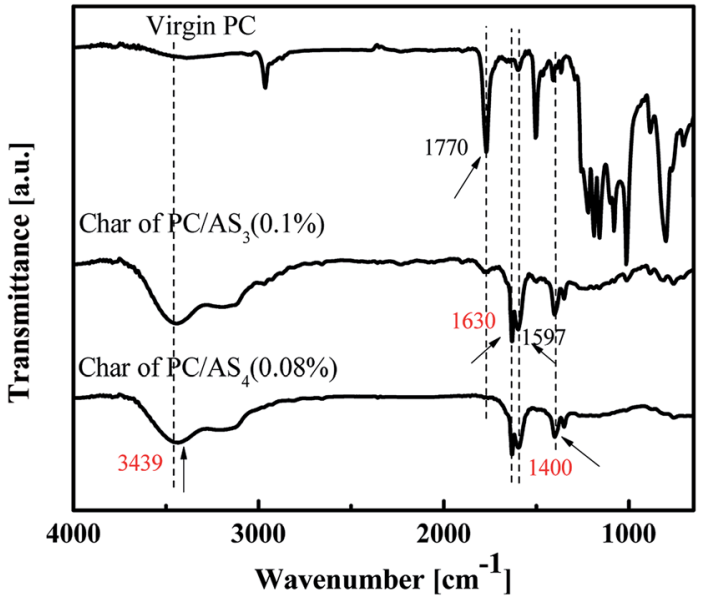

Fig. 9 FT-IR spectra of virgin $\mathrm{PC}$, and the residual char of $\mathrm{PC} / \mathrm{AS}_{3}$ $(0.1 \%)$ and $\mathrm{PC} / \mathrm{AS}_{4}(0.08 \%)$.

vibration of $-\mathrm{OH}$ groups, respectively. The characteristic peaks in $1630 \mathrm{~cm}^{-1}$ and $1597 \mathrm{~cm}^{-1}$ are attributed to the $-\mathrm{C}=\mathrm{C}-$ in the benzene ring. The above information in FT-IR spectra of residue char for $\mathrm{PC} \mathrm{AS}_{n}(n=3,4)$ composites indicate that some aromatic alcohol compounds are produced in the residual char of $\mathrm{PC} / \mathrm{AS}_{3}$ or $\mathrm{PC} / \mathrm{AS}_{4}$ after combustion.

From the FT-IR results in combination with the above char morphology and TG analysis of PC and its composites, we can obtain the flame retarding mechanism as follows: the flame retardant $\mathrm{AS}_{3}$ or $\mathrm{AS}_{4}$ first decomposes in case of fire and produces $\mathrm{SO}_{2}$ that serves as a kind of catalyst to accelerate the decomposition of PC. Subsequently, some compounds containing aromatic alcohol are then generated and further crosslinked automatically to promote intumescent char formation, so that the unburned PC is protected with high flame retardancy.

\section{Conclusions}

Two flame retardants $\left(\mathrm{AS}_{3}\right.$ and $\left.\mathrm{AS}_{4}\right)$ with multi-sulfonate centered on adamantane have been synthesized successfully and characterized by ${ }^{1} \mathrm{H}$ NMR and FTIR spectra. The TGA results of $\mathrm{AS}_{3}$ and $\mathrm{AS}_{4}$ demonstrate that the novel flame retardants have matchable thermal stability with PC matrix. Both the ternary sulfonate $\mathrm{AS}_{3}$ and the quaternary sulfonate $\mathrm{AS}_{4}$ exhibit high flame retardant ability to PC. Only $0.1 \mathrm{wt} \% \mathrm{AS}_{3}$ or $0.08 \mathrm{wt} \% \mathrm{AS}_{4}$ can provide $\mathrm{PC}$ composites with UL-94 V-0 rating and improve the LOI value from $25.8 \%$ to $31.2 \%$ and $32.3 \%$ for $\mathrm{PC}^{-\mathrm{AS}_{3}}(0.1 \%)$ and $\mathrm{PC} / \mathrm{AS}_{4}(0.08 \%)$, respectively. Moreover, the results from cone calorimetry show that HRR, THR and COY of both PC/AS and $\mathrm{PC} / \mathrm{AS}_{4}$ are reduced compared to the neat PC. In addition, they can maintain good mechanical strength, especially for $\mathrm{PC} / \mathrm{AS}_{4}$ which displays increased mechanical strength in most cases. Finally, we obtain the flame retarding mechanism of this new $\mathrm{PC} / \mathrm{AS}_{n}$ system is that $\mathrm{AS}_{3}$ and $\mathrm{AS}_{4}$ have a high catalytic effect to accelerate $\mathrm{PC}$ thermal degradation and protective char formation. This novel sulfonate flame retardant system provide a new green solution for decreasing the deterioration of the mechanical performance and enhancing the flame retardancy simultaneously.

\section{Acknowledgements}

The authors would like to thank Sun Yat-sen University for plastic processing equipments, Kingfa Sci. and Tech. Co., Ltd. for supporting cone calorimetry tests. In addition, we thank James Wilk who is a English teacher in University of Massachusetts-Amherst for helping us to check the English writing of the revised manuscript. Funding: This work was supported by the National Natural Science Foundation of China [grant numbers 21476051, 21506038 and 21676058], the Nature Science Foundation of Guangdong Province [grant number 2014A030310307], and the Science and Technology Project of Guangdong Province [grant numbers 2013B010403028 and 2016A050502057].

\section{References}

1 S. Bourbigot and S. Duquesne, J. Mater. Chem., 2007, 17, 2283-2300.

2 S. Y. Lu and I. Hamerton, Prog. Polym. Sci., 2002, 27, 16611712.

3 C. Hoffendahl, G. Fontaine, S. Duquesne, F. Taschner, M. Mezgerb and S. Bourbigot, RSC Adv., 2014, 4, 2018520199.

4 R. Jeencham, N. Suppakarn and K. Jarukumjorn, Composites, Part B, 2014, 56, 249-253.

5 C. C. Xi, G. Z. Kang, F. C. Lu, J. W. Zhang and H. Jiang, Mater. Des., 2015, 67, 644-648.

6 S. V. Levchik and E. D. Weil, Polym. Int., 2005, 54, 981-998.

7 S. V. Levchik and E. D. Weil, J. Fire Sci., 2006, 24, 137-151.

8 B. Swoboda, S. Buonomo, E. Leroy and J. M. Cuesta Lopez, Polym. Degrad. Stab., 2008, 93, 910-917. 
9 Y. H. Guan, W. Liao, Z. Z. Xu, M. J. Chen, J. Q. Huang and Y. Z. Wang, RSC Adv., 2015, 5, 59865-59873.

10 Q. X. He, L. Tang, T. Fu, Y. Q. Shi, X. L. Wang and Y. Z. Wang, RSC Adv., 2016, 6, 52485-52494.

11 T. Yu, T. Tuerhongjiang, C. Sheng and Y. Li, Composites, Part A, 2017, 97, 60-66.

12 M. Rajaei, D. Y. Wang and D. Bhattacharyya, Composites, Part $B$, 2017, 113, 381-390.

13 L. Dang, X. Nai, Y. Dong and W. Li, RSC Adv., 2017, 7, 2165521665.

14 W. C. Zhang, X. M. Li, H. B. Fan and R. J. Yang, Polym. Degrad. Stab., 2012, 97, 2241-2248.

15 J. P. Ding, Z. Q. Tao, X. B. Zuo, L. Fan and S. Y. Yang, Polym. Bull., 2009, 62, 829-841.

16 X. P. Hu, Y. Y. Guo, L. Chen, X. L. Wang, L. J. Li and Y. Z. Wang, Polym. Degrad. Stab., 2012, 97, 1772-1778.

17 L. Li, P. Wei, J. Li, J. Jow and K. Su, J. Fire Sci., 2010, 28, 523538.

18 Z. Hu, L. Chen, B. Zhao, Y. Luo, D. Y. Wang and Y. Z. Wang, Polym. Degrad. Stab., 2011, 96, 320-327.

19 R. K. Jian, L. Chen, Z. Hu and Y. Z. Wang, J. Appl. Polym. Sci., 2012, 123, 2867-2874.

20 W. Zhao, B. Li, M. Xu, K. Yang and L. Lin, Fire Mater., 2013, 37, 530-546.
21 K. H. Pawlowski and B. Schartel, Polym. Int., 2007, 56, 14041414.

22 L. Duan, H. Yang, L. Song, Y. Hou, W. Wang, Z. Gui and Y. Hu, Polym. Degrad. Stab., 2016, 134, 179-185.

23 Z. J. Jiang, S. M. Liu, J. Q. Zhao and X. B. Chen, Polym. Degrad. Stab., 2013, 98, 2765-2773.

24 S. Q. Fu, J. W. Guo, D. Y. Zhu, Z. Yang, C. F. Yang, J. X. Xian and X. Li, RSC Adv., 2015, 5, 67054-67065.

25 S. B. Nie, Y. Hu, L. Song, S. Q. He and D. D. Yang, Polym. Adv. Technol., 2008, 19, 489-495.

26 Y. Z. Wang, B. Yi, B. Wu, B. Yang and Y. Liu, J. Appl. Polym. Sci., 2003, 89, 882-889.

27 A. Nodera and T. Kanai, J. Appl. Polym. Sci., 2004, 94, 21312139.

28 S. M. Liu, H. Ye, Y. S. Zhou, J. H. He, Z. J. Jiang, J. Q. Zhao and X. B. Huang, Polym. Degrad. Stab., 2006, 91, 1808-1814.

29 H. Newman, Synthesis, 1972, 12, 692-693.

30 A. Genovese and R. A. Shanks, Composites, Part A, 2008, 39, 398-405.

31 W. Y. Xing, W. Yang, W. J. Yang, Q. H. Hu, J. Y. Si, H. D. Lu, B. H. Yang, L. Song, Y. Hu and R. K. K. Yuen, ACS Appl. Mater. Interfaces, 2016, 39, 26266-26274.

32 Q. X. He, L. Tang, T. Fu, Y. Q. Shi, X. L. Wang and Y. Z. Wang, RSC Adv., 2016, 6, 52485-52494. 